\title{
Interaction of prostate specific membrane antigen with clathrin and the adaptor protein complex-2
}

\author{
OSCAR B. GOODMAN Jr ${ }^{1,2}$, SONALI P. BARWE ${ }^{3}$, BRIGITTE RITTER ${ }^{4}$, PETER S. McPHERSON ${ }^{4}$, \\ ANN-JEANETTE VASKO ${ }^{5}$, JAMES H. KEEN ${ }^{5}$, DAVID M. NANUS ${ }^{1,2}$, \\ NEIL H. BANDER ${ }^{1}$ and AYYAPPAN K. RAJASEKARAN ${ }^{3}$
}

\begin{abstract}
${ }^{1}$ Urologic Oncology Research Laboratory, Department of Urology, and ${ }^{2}$ Division of Hematology and Medical Oncology, Department of Medicine, Weill Medical College of Cornell University-New York Presbyterian Hospital, New York, NY 10021; ${ }^{3}$ Department of Pathology and Laboratory Medicine, David Geffen School of Medicine, University of California, Los Angeles, CA 90095, USA; ${ }^{4}$ Department of Neurology and Neurosurgery, Montreal Neurological Institute, McGill University, 3801 University, Montreal, QC, H3A 2B4, Canada; ${ }^{5}$ Kimmel Cancer Institute, Jefferson Medical College of Thomas Jefferson University, Philadelphia, PA 19102, USA
\end{abstract}

Received February 20, 2007; Accepted April 13, 2007

\begin{abstract}
Prostate-specific membrane antigen (PSMA) is an integral membrane glycoprotein expressed in prostatic epithelia and is being evaluated as a therapeutic target in prostate cancer. It undergoes constitutive receptor-mediated endocytosis via clathrin-coated pits, which is enhanced in the presence of monoclonal antibodies directed against it. We describe distinct interactions of PSMA with clathrin and the clathrin adaptor protein-2 (AP-2) complex, two components of clathrin-coated pits. The intracellular N-terminal domain of PSMA interacts with the N-terminal globular domain of clathrin heavy chain. Deletion analysis revealed an important determinant of this interaction residing within the proximal portion of the clathrin heavy chain $\mathrm{N}$-terminal domain (amino acids 1-85) distinct from the clathrin binding sites of other known clathrin-binding proteins. Furthermore, PSMA interacts with the ear domain of $\alpha$-adaptin (an AP-2 subunit), and a glutamic acid residue at position 7 in the cytoplasmic tail of PSMA is essential for this interaction. These data indicate that PSMA exhibits a high affinity, specific association with the clathrin-based endocytic machinery by distinct interactions with both clathrin and AP-2. Thus, although PSMA is a new member of the dual AP and clathrin binding proteins, its $\alpha$-adaptin and clathrin heavy chain binding determinants are distinct from those of other members.
\end{abstract}

Correspondence to: Dr Ayyappan K. Rajasekaran, Department of Pathology and Laboratory Medicine, Room 13-344 CHS, University of California, Los Angeles, CA 90095, USA

E-mail: arajasekaran@mednet.ucla.edu

Key words: prostate cancer, prostate specific membrane antigen, clathrin, endocytosis

\section{Introduction}

Prostate specific membrane antigen (PSMA) is a type II transmembrane protein (1-3). It comprises a 19 amino acid Nterminal intracellular domain, a single transmembrane domain of 24 amino acids, and a 707 amino acid ectodomain, sharing modest degrees of homology with the transferrin receptor $(2,4)$. PSMA exists as a homo-dimer at the cell surface (5). The extracellular domain of PSMA is highly glycosylated, and possesses both folate hydrolase and $\mathrm{N}$-acetylated, $\alpha$-linked acidic dipeptidase (NAALADase) activities. These enzymatic activities are enhanced in metastatic prostate cancer (6). The expression of PSMA is highly restricted in normal tissue, predominantly to prostatic epithelium, with low expression in brain, kidney, salivary gland, and duodenum. Its function in the prostate remains unknown, but its expression correlates with prostate cancer clinical stage (7). These correlative data have led investigators to hypothesize that PSMA is an attractive target for novel anti-neoplastics in the form of either naked or cargo-coupled monoclonal antibodies (mAbs). PSMA is also expressed on endothelial cells of malignant neovasculature in a variety of solid tumors, making it a potential target for antiangiogenic therapy in these diseases (8).

The intracellular N-terminal domain of PSMA plays a critical role in its internalization and subcellular localization. Previously, our group demonstrated that the cytoplasmic tail of PSMA interacts with filamin A, an actin crosslinking protein, and this interaction seems to be involved in PSMA localization to the recycling endosomal compartment (9). Like transferrin receptor, PSMA is known to undergo constitutive endocytosis via clathrin-coated pits. This internalization is enhanced approximately 3-fold by monoclonal antibodies directed against the ectodomain of PSMA (10). Recent work has demonstrated a role for a novel methionine-leucine based MXXXL motif located at the N-terminus of the protein in targeting PSMA for internalization, with residues Met-1 and Leu-5 playing a critical role (11). 
The ability to modulate internalization of PSMA is important for maximizing therapeutic efficacy of mAbs, i.e. antibodydependent cellular cytotoxicity. Alternatively, cytotoxic cargos affixed to the mAb could be more effectively delivered to an intracellular compartment if the rate of mAb endocytosis were enhanced. In order to develop selective modulators of PSMA internalization, the molecular determinants governing the interaction of PSMA with the clathrin-based endocytic machinery need to be identified. In this study, we demonstrate that like other proteins internalized via clathrin-coated pits, PSMA interacts with both, clathrin and the clathrin adaptor protein-2 (AP-2) ear domain of the plasma membrane clathrinbased endocytic machinery.

\section{Materials and methods}

Antibodies. The mouse mAb J591 against the extracellular domain of PSMA has been described earlier (12). The mAb 7E11 directed against the cytoplasmic domain of PSMA was prepared from hybridoma 7E11 (American Type Culture Collection). Horseradish peroxidase-conjugated anti-mouse antibody was purchased from BD Transduction Laboratories.

Cell culture. MDCK cells (clone II) expressing either WTPSMA (MDCK-PSMA) (13) or Glu-7-Ala mutant of PSMA (MDCK-PSMA $_{\mathrm{Ala} 7}$ ) (to be published elsewhere) were cultured in DMEM supplemented with $10 \%$ (v/v) fetal bovine serum, $2 \mathrm{mM}$ L-glutamine, $25 \mathrm{U} / \mathrm{ml}$ penicillin, $25 \mu \mathrm{g} / \mathrm{ml}$ streptomycin, and $100 \mu \mathrm{M}$ non-essential amino acids. Cells were grown at $37^{\circ} \mathrm{C}$ in a humidified incubator with $5 \%(\mathrm{v} / \mathrm{v}) \mathrm{CO}_{2}$, and were treated for $6 \mathrm{~h}$ in media containing $10 \mathrm{mM}$ sodium butyrate (Sigma) to increase PSMA expression, prior to lysis.

Cage and coat binding assays. Preparation of preformed cages and clathrin coats was performed as described previously (14). Recombinant PSMA (rPSMA) was overexpressed and purified from sf9 insect cells as previously described (15). Binding to clathrin substrates was performed as previously described (16). Briefly, $300 \mathrm{nM}$ rPSMA was incubated with clathrin preparations (100 $\mathrm{nM}$ trimers) in 0.1 $\mathrm{M}$ Na-MES, pH 6.8, $0.1 \mathrm{mM}$ dithiothreitol, $0.1 \mathrm{mg} / \mathrm{ml}$ soybean trypsin inhibitor (buffer A) for $10 \mathrm{~min}$ at room temperature. Complexes were centrifuged through a $75 \mathrm{ml} 0.2 \mathrm{M}$ sucrose cushion prepared in buffer $\mathrm{A}$ in a TLA100 rotor (Beckman) at 75,000 rpm for $5 \mathrm{~min}$ at $4^{\circ} \mathrm{C}$ and analyzed by Western blotting using mAb J591.

GST-clathrin binding assays. GST-clathrin fusion proteins were prepared as previously described (16). Briefly, GST-clathrin $(100 \mathrm{nM})$ and rPSMA $(10 \mathrm{nM})$ were incubated in a final volume of $300 \mu 1$ using a $10 \mu \mathrm{l}$ bed volume of beads for $30 \mathrm{~min}$ on a rotator in a buffer containing $20 \mathrm{mM} \mathrm{K-HEPES,} \mathrm{pH} \mathrm{7.3,}$ $120 \mathrm{mM}$ potassium acetate, $0.1 \mathrm{mM}$ dithiothreitol, $0.1 \mathrm{mg} / \mathrm{ml}$ soybean trypsin inhibitor, $1 \mu \mathrm{g} / \mathrm{ml}$ each leupeptin, pepstatin, and antipain, and $0.1 \%(\mathrm{v} / \mathrm{v})$ Triton X-100. The samples were washed twice with $1 \mathrm{ml}$ of buffer, eluted with SDS-PAGE sample buffer, and equal aliquots were analyzed by Western blotting using mAb J591.

$\alpha$-adaptin ear binding assays. The WT ear domain of $\alpha$-adaptin subunit of the AP-2 complex (aa701-938), and its mutant
(Gln-782-Ala), which abolishes ear domain interaction with tryptophan-phenylalanine based WXXF-acidic motifs, cloned in pGEX-4T1 are described earlier $(17,18)$. Purification of GST-fusion proteins was performed as described previously (17-19). MDCK-PSMA or MDCK-PSMA ${ }_{\mathrm{Ala} 7}$ cells grown to $70-80 \%$ confluence were lysed in a buffer containing $20 \mathrm{mM}$ Tris- $\mathrm{HCl}, \mathrm{pH} 7.4,100 \mathrm{mM} \mathrm{NaCl}, 1 \%$ Triton X-100, $1 \mathrm{mM}$ EDTA, $1 \mathrm{mM}$ EGTA, $1 \mathrm{mM}$ sodium glycerolphosphate, $1 \mathrm{mM}$ sodium orthovanadate, $1 \mathrm{mM}$ PMSF, and $5 \mu \mathrm{g} / \mathrm{ml}$ each of antipain, leupeptin, and pepstatin. MDCK-PSMA $\mathrm{Ala}_{7}$ cells were additionally treated with $10 \mu \mathrm{g} / \mathrm{ml}$ of leupeptin for $1 \mathrm{~h}$ prior to lysis. The lysates were sonicated three times for $10 \mathrm{sec}$ each, and clarified by centrifugation at $16,000 \times \mathrm{g}$ at $4^{\circ} \mathrm{C}$ for $10 \mathrm{~min}$. Total protein was estimated from the supernatants using the Bio-Rad DC reagent as per the manufacturer's instructions. Lysates normalized to the expression levels of WT and mutant PSMA were incubated with either GST alone or GST-fusion proteins at $4^{\circ} \mathrm{C}$, overnight with constant agitation. To show equal amounts of PSMA in the input, PSMA was immunoprecipitated by using mAb J591 from lysates corresponding to $10 \%$ of the amount of lysates used for GST pulldown assay. After incubation, the beads were washed three times with lysis buffer, resolved on an SDS-PAGE, and immunoblotted with the indicated antibodies.

\section{Results}

PSMA interacts with clathrin and AP-2 containing substrates. To characterize the interaction between PSMA and the clathrinbased endocytic machinery, we incubated PSMA in the presence or absence of co-assembled pure clathrin coats composed of purified clathrin and AP-2 (14), and analyzed high speed sedimentation fractions for the presence of PSMA by a Western blot using mAb J591, followed by densitometry (Fig. 1A). In the presence of clathrin coats $34.50 \pm 0.01 \%$ of the PSMA specifically sedimented, reflecting binding of PSMA to clathrin coats (Fig. 1, upper panel, lane 3 versus lane 2). As a negative control, we utilized recombinant neprilysin, another cell surface peptidase not known to internalize via clathrin-coated pits (20), and observed $0.29 \pm 0.12 \%$ specific sedimentation, likely reflecting nonspecific trapping by coat lattices. No endogenous NEP or PSMA was observed in the coat structures (Fig. 1, lane 4).

Next, we determined whether PSMA directly interacts with clathrin by assessing for sedimentation using increasing concentrations of preformed pure clathrin cages that lack AP-2 and the association was evaluated by Western blotting of the co-sedimented PSMA. As shown in Fig. 1B, PSMA binding to clathrin cages was saturable, and of high affinity, exhibiting a $\mathrm{Kd}$ of $24.8 \mathrm{nM}$, with a calculated Bmax of $18.6 \%$ of the input. These results indicate that PSMA specifically associates with clathrin.

The N-terminal domains of the clathrin triskelion project towards the plasma membrane. This orientation enables them to interact with transmembrane proteins such as the low density lipoprotein (21), and adaptors such as $B$ arrestins (16) and AP-2 (22). Therefore, we assessed whether PSMA also interacts with the N-terminal domain of clathrin by using a glutathione-S-transferase fused to the first 579 amino acids of clathrin (GST-clathrin) immobilized on glutathione-agarose 
A

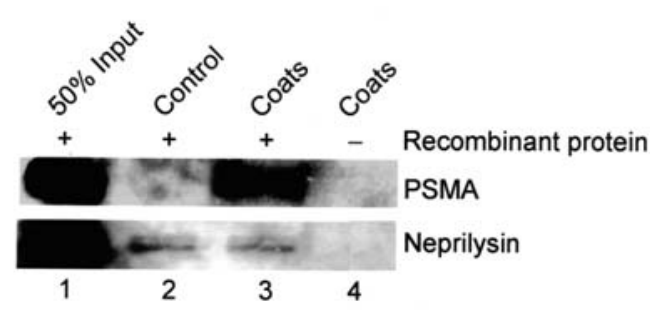

B

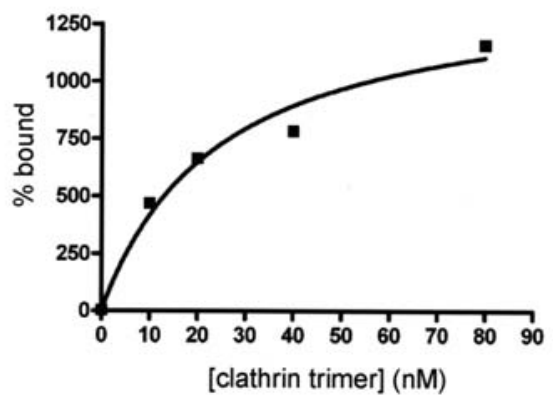

Figure 1. Binding of rPSMA to native clathrin substrates. (A) rPSMA or recombinant neprilysin $(300 \mathrm{nM})$ was incubated in the absence (control) or presence of co-assembled clathrin coats (100 $\mathrm{nM}$ clathrin trimers) for $10 \mathrm{~min}$ at room temperature, and aliquots of high speed sediments analyzed by Western blotting using mAb J591 (PSMA, upper panel) or $\mathrm{NCl}$ antibodies (neprilysin, lower panel). Lane 1 represents a 50\% input standard, and lane 4 represents endogenous proteins contained within the coat structures. Bands were quantified densitometrically using imageJ software $(\mathrm{NIH})$ and expressed in the text as mean \pm SEM for duplicate values. Results are representative of two independent experiments. (B) rPSMA $(10 \mathrm{nM})$ was incubated with the indicated concentration of clathrin trimer as preformed clathrin cages and subjected to high speed sedimentation and Western blotting using mAb J591. Bands were quantified densitometrically using imageJ software (NIH). Results are representative of three independent experiments.

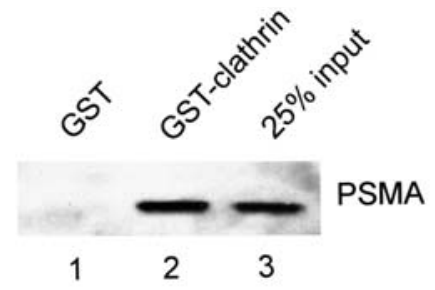

Figure 2. Binding of rPSMA to recombinant clathrin N-terminal domain. rPSMA (10 nM) was incubated with GST alone (lane 1) or GST-clathrin heavy chain (1-579) (lane 2) bound to glutathione-agarose beads, and following washing with $1 \mathrm{ml}$ of binding buffer, SDS-sample buffer eluants were analyzed by SDS-PAGE followed by Western blotting for PSMA with mAb J591. Lane 3 corresponds to $25 \%$ of the total rPSMA input. Results are representative of two independent experiments.

beads, or as a control, GST alone. As shown in Fig. 2, >25\% of the input protein specifically bound to GST-clathrin containing beads, but none was detectable on the GST beads, indicating that a specific PSMA binding site resides on the N-terminal domain of the clathrin heavy chain.

Mapping of the PSMA-binding site on clathrin heavy chain. Clathrin heavy chain N-terminal domain consists of seven propeller blades (Fig. 3A), each formed by four anti-parallel $\beta$-sheets (a-d), followed by a series of $11 \alpha$ helices $(\alpha 1-\alpha 11)$. The $7 \mathrm{~d} B$-sheet comprises amino acids 1-23 and is followed
A

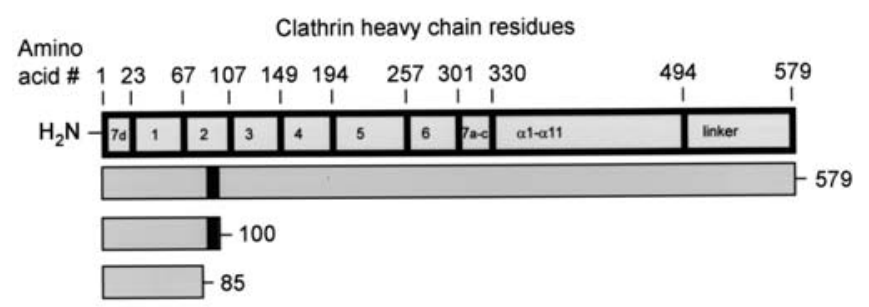

B

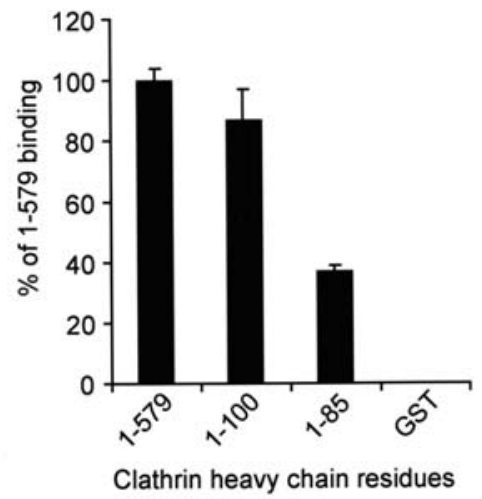

Figure 3. Deletion analysis of rPSMA binding to clathrin N-terminal domain. (A) Domain structure of N-terminal domain of clathrin heavy chain. B-rich propeller blade structures, with blades numbered 1-7; are followed by the $\alpha$-helical domain ( $\alpha 1-\alpha 11)$, and the N-terminal domain linker residues (linker). The residues corresponding to the four constructs tested for PSMA binding are aligned against the upper panel. The canonical clathrin box-binding region lies within residues 85-100 contained in the second propeller blade (black bar, see text for details). (B) rPSMA was incubated with GST fused to the indicated clathrin heavy chain residues pre-fixed to glutathione-agarose beads, and following washing with $1 \mathrm{ml}$ of binding buffer, SDS-sample buffer eluants were analyzed by SDS-PAGE followed by Western blotting for PSMA with $\mathrm{mAb}$ J591. Bands were quantified densitometrically using imageJ software $(\mathrm{NIH})$ and error bars denote the mean \pm the standard error of triplicate bands. Binding is expressed relative to that of GST-clathrin heavy chain (1-579), the full length N-terminal domain. Results are representative of two independent experiments.

sequentially by blades $1-6$, and $\beta$ sheets 7 a-c (amino acids 24-330). The canonical clathrin box binding sequence resides in the middle of the second blade situated in the diagonal groove interfaced with the first blade $(16,23)$. A series of GST-clathrin heavy chain fusion proteins were utilized to identify the clathrin residues that interact with PSMA. Binding to the first 100 amino acids of clathrin was equivalent to that of the first 579 amino acids (Fig. 3B), corresponding to the entire N-terminal domain of clathrin. Interestingly, there was still significant PSMA binding function retained by the first 85 amino acids, indicating that a distinct binding determinant is proximal to the region of clathrin known to interact with other classic clathrin box motifs (16).

Interaction of the ear domain of $\alpha$-adaptin subunit of AP-2 complex with PSMA. Recent studies have identified a tryptophan-phenylalanine based WXXF-acidic motif involved in binding of accessory proteins to the globular ear domain of the $\alpha$-adaptin subunit of the AP- 2 complex ( $\alpha$-ear). An acidic residue at position +5 where residue Trp is designated as 0 position, found in other $\alpha$-ear binding proteins has shown 
A

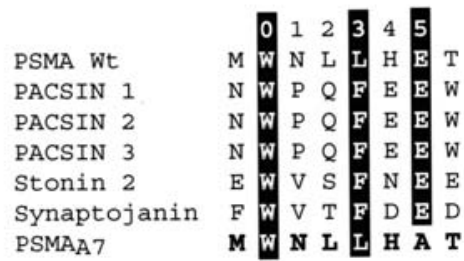

B

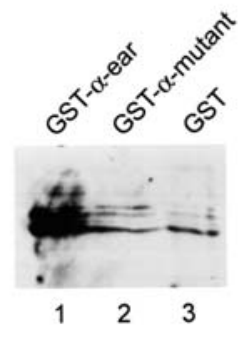

C

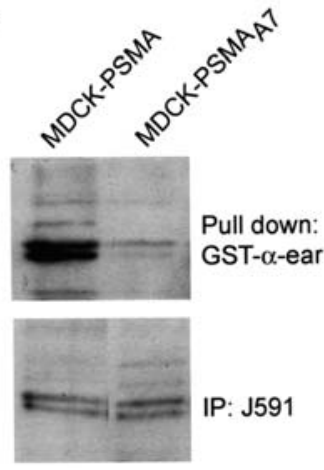

Figure 4. PSMA binding to the AP-2 $\alpha$-adaptin ear domain. (A) Alignment of PSMA N-terminus with motifs from known AP- $2 \alpha$-adaptin ear domain binding proteins. Position 0 denotes the conserved tryptophan residue (see text for details). (B) Glutathione-sepharose beads pre-coupled to GST alone, or GST fusion proteins encoding either the WT (GST- $\alpha$-ear) or the Gln-782Ala mutant (GST- $\alpha$-mutant) ear domains of AP- $2 \alpha$-adaptin were incubated with lysates of MDCK cells expressing WT-PSMA. The bound PSMA was detected by immunoblotting with mAb J591. (C) MDCK cells expressing either WT-PSMA (MDCK-PSMA) or the Glu-7-Ala mutant of PSMA $\left(\right.$ MDCK-PSMA $\left._{\mathrm{Ala} 7}\right)$ were lysed, and incubated with either GST- $\alpha$-ear bound to glutathione-sepharose beads or mAb J591-coupled agarose beads. PSMA pulled down was detected by immunoblotting with mAb 7E11. For immunoprecpitation with mAb J591, 10\% of the input for GST pull-down assay was used.

to be essential for $\alpha$-ear binding (18). The endocytic motif in the cytoplasmic tail of PSMA (Met-Trp-Asn-Leu-Leu) contains the conserved WXXF based motif and an acidic residue at position +5 (Fig. 4A), suggesting that PSMA might bind to the $\alpha$-ear via its endocytic motif. To test this possibility, we performed a GST-pull down assay using the $\alpha$-ear expressed as a GST-fusion protein (GST- $\alpha$-ear). Wild-type PSMA stably expressed in the epithelial cell line MDCK (13) affinity precipitated GST- $\alpha$-ear, but not GST alone (Fig. 4B, compare lanes 1 and 3). Mutation of the $\alpha$-ear residue Gln-782, previously shown to be critical in interactions with other endocytic proteins bearing WXXF-acidic motifs (17), completely abrogated binding to PSMA (Fig. 4B, lane 2). The mutation of the acidic residue $(\mathrm{Glu})$ at position +5 to $\mathrm{Ala}\left(\mathrm{PSMA}_{\mathrm{Ala} 7}\right)$ impaired $\alpha$-ear binding to PSMA (Fig. 4C), indicating that the acidic residue at position +5 is important for PSMA interaction with $\alpha$-ear. No interaction was seen with other known adaptor binding regions of AP-2 including $\beta$-adaptin and $\mu$ subunits (data not shown). These results indicate that PSMA interaction with the $\alpha$-ear is through the WXXF acidic motif present on the cytoplasmic tail of PSMA.

\section{Discussion}

In this study, we have identified independent interactions between PSMA and both clathrin and AP-2. We showed that

co-assembled, pure clathrin-coats bind PSMA but not neprilysin, a protease that does not undergo clathrin-dependent internalization. In addition, in vitro affinity precipitation experiments clearly reveal PSMA binding to GST-clathrin but not to GST alone. Furthermore, the saturation kinetics of PSMA interaction with clathrin, and different levels of clathrin heavy chain truncation mutants binding to PSMA, strongly suggest that PSMA interaction with clathrin is specific. The specificity of PSMA binding to AP-2 was established by two complementary approaches: i) PSMA interacts with only wild type $\alpha$-ear but not the mutant form that does not associate with other endocytic proteins; and ii) the mutation of the acidic residue at +5 position of PSMA abrogates its interaction with $\alpha$-ear. These data suggest that PSMA can interact with the endocytic machinery with high affinity and specificity, serving to target PSMA for internalization.

PSMA is a new member of the growing family of dual AP and clathrin binding proteins such as $\beta$-arrestins (16), auxillins $(24,25)$, and Huntingtin interacting protein-1 (HIP1) $(26,27)$. With regard to other known dual clathrin and AP-2 binding proteins, PSMA interacts with a discrete region on the clathrin molecule, although it may share some determinants common to other known clathrin binding proteins. $\beta$-arrestins, for example, interact principally with residues 86-100 on clathrin N-terminal domain. While this region does interact with PSMA, it is not the exclusive determinant, as the first 85 amino acids of clathrin heavy chain retain an ability to interact with PSMA. Based on the propeller blade-like crystal structure of the clathrin N-terminal domain (23), the PSMA binding site on clathrin resides on the proximal portion of propeller blade 2 and/or blade 1 (Fig. 3B). Interestingly, PSMA possesses a non-classical clathrin box (Leu-His-Glu-ThrAsp), juxtaposed to the endocytic motif (Met-Trp-Asn-LeuLeu). The observed greater extent of binding to co-assembled coat structures containing both clathrin and AP-2 (Fig. 1A) suggests that these two domains are distinct, and is further supported by the observation that the first 85 amino acids can support PSMA binding, a region of clathrin not previously shown to interact with other classic clathrin box containing proteins. Although clathrin terminal domain is a major PSMA binding region, our data do not exclude the possibility of interactions with other regions of the clathrin molecule.

While non-visual arrestins interact with the $\beta_{2}$-adaptin subunit of AP-2 molecule (28), PSMA interacts with the ear domain of $\alpha$-adaptin subunit of AP-2 complex. The multiple differing molecular interactions of dual AP-clathrin binding proteins reflect the growing combinatorial diversity of the endocytic compartment's molecular interactions with potential cargos, and may also serve to regulate interactions between the actin cytoskeleton and the endocytic vesicles. PSMA also associates with filamin and this binding modulates PSMA internalization and enzyme activity (9). Similarly, non-visual arrestins, interact with clathrin and AP-2 following agonist, and also bind filamin A, and this binding is disrupted by receptor agonist (29). Like PSMA, HIP1 interacts with the appendage domain of $\alpha$-adaptin and clathrin (27) and coincidently, is also overexpressed in androgen-independent prostate cancer (30), suggesting a functional interaction between these two proteins. However, HIP1 uses phenylalanine-aspartate based FXDXF motifs to engage the platform sub-domain of the $\alpha$-ear, whereas 
PSMA uses the WXXF-acidic binding site on the sandwich sub-domain of $\alpha$-ear, suggesting that there is a minimal overlap between the domains of $\alpha$-ear that interact with PSMA and HIP1.

Therapeutically, PSMA represents an important antineoplastic target given its restricted cell surface expression. With regard to prostate cancer, it is an ideal target because its expression increases with cancer progression. More recently, PSMA has been noted to be expressed on malignant neovasculature to varying degrees, most notably in renal cancers and glioblastoma multiforme (8), but not normal vasculature, suggesting that it may be an outstanding target for antiangiogenic therapy as well. PSMA undergoes constitutive internalization via clathrin-coated pits in a manner similar to transferrin receptor. In the presence of $\mathrm{mAb}$ directed against PSMA, this rate of internalization increases $>3$-fold, a rationale for affixing cytotoxic cargoes to mAbs. Thus, understanding the molecular interactions governing the interaction of PSMA with the endocytic machinery represents an important prerequisite for enhancing the potency of anti-PSMA therapy. For instance, one would predict that antibody dependent cell-mediated cytotoxicity of naked mAb might be augmented by inhibiting PSMA endocytosis, while increasing internalization could facilitate intracellular delivery of cytotoxic cargos. The phenomenon that PSMA expression is increased in advanced prostate cancer suggests that PSMA expression may impart a selective growth advantage through stimulation of mitogenic signaling pathways, which may be highly dependent on compartmentalization.

\section{Acknowledgements}

This work was supported by Sass Foundation for Cancer Research and McCooey Cancer Research Fund (O.B.G.) and DOD W81XWH-04-1-0113 (A.K.R.).

\section{References}

1. Heston WD: Characterization and glutamyl preferring carboxypeptidase function of prostate specific membrane antigen: a novel folate hydrolase. Urology 49: 104-112, 1997.

2. Israeli RS, Powell CT, Fair WR and Heston WD: Molecular cloning of a complementary DNA encoding a prostate-specific membrane antigen. Cancer Res 53: 227-230, 1993.

3. Pinto JT, Suffoletto BP, Berzin TM, et al: Prostate-specific membrane antigen: a novel folate hydrolase in human prostatic carcinoma cells. Clin Cancer Res 2: 1445-1451, 1996.

4. Mahadevan D and Saldanha JW: The extracellular regions of PSMA and the transferrin receptor contain an aminopeptidase domain: implications for drug design. Protein Sci 8: 2546-2549, 1999.

5. Schulke N, Varlamova OA, Donovan GP, et al: The homodimer of prostate-specific membrane antigen is a functional target for cancer therapy. Proc Natl Acad Sci USA 100: 12590-12595, 2003.

6. Lapidus RG, Tiffany CW, Isaacs JT and Slusher BS: Prostatespecific membrane antigen (PSMA) enzyme activity is elevated in prostate cancer cells. Prostate 45: 350-354, 2000.

7. Murphy GP, Elgamal AA, Su SL, Bostwick DG and Holmes EH: Current evaluation of the tissue localization and diagnostic utility of prostate specific membrane antigen. Cancer 83: 2259-2269, 1998.

8. Chang SS, Reuter VE, Heston WD, Bander NH, Grauer LS and Gaudin PB: Five different anti-prostate-specific membrane antigen (PSMA) antibodies confirm PSMA expression in tumor-associated neovasculature. Cancer Res 59: 3192-3198, 1999.

9. Anilkumar G, Rajasekaran SA, Wang S, Hankinson O, Bander NH and Rajasekaran AK: Prostate-specific membrane antigen association with filamin A modulates its internalization and NAALADase activity. Cancer Res 63: 2645-2648, 2003.
10. Liu H, Rajasekaran AK, Moy P, et al: Constitutive and antibodyinduced internalization of prostate-specific membrane antigen. Cancer Res 58: 4055-4060, 1998.

11. Rajasekaran SA, Anilkumar G, Oshima E, et al: A novel cytoplasmic tail MXXXL motif mediates the internalization of prostate-specific membrane antigen. Mol Biol Cell 14: 48354845, 2003.

12. Liu H, Moy P, Kim S, et al: Monoclonal antibodies to the extracellular domain of prostate-specific membrane antigen also react with tumor vascular endothelium. Cancer Res 57: 36293634, 1997.

13. Christiansen JJ, Rajasekaran SA, Moy P, et al: Polarity of prostate specific membrane antigen, prostate stem cell antigen, and prostate specific antigen in prostate tissue and in a cultured epithelial cell line. Prostate 55: 9-19, 2003.

14. Zaremba S and Keen JH: Assembly polypeptides from coated vesicles mediate reassembly of unique clathrin coats. J Cell Biol 97: 1339-1347, 1983.

15. Lodge PA, Childs RA, Monahan SJ, et al: Expression and purification of prostate-specific membrane antigen in the baculovirus expression system and recognition by prostate-specific membrane antigen-specific T cells. J Immunother 22: 346-355, 1999.

16. Goodman OB Jr, Krupnick JG, Gurevich VV, Benovic JL and Keen JH: Arrestin/clathrin interaction. Localization of the arrestin binding locus to the clathrin terminal domain. J Biol Chem 272: 15017-15022, 1997.

17. Ritter B, Denisov AY, Philie J, Deprez C, Tung EC, Gehring K and McPherson PS: Two WXXF-based motifs in NECAPs define the specificity of accessory protein binding to AP-1 and AP-2. EMBO J 23: 3701-3710, 2004.

18. Ritter B, Philie J, Girard M, Tung EC, Blondeau F and McPherson PS: Identification of a family of endocytic proteins that define a new alpha-adaptin ear-binding motif. EMBO Rep 4: 1089-1095, 2003.

19. Barwe SP, Anilkumar G, Moon SY, Zheng Y, Whitelegge JP, Rajasekaran SA and Rajasekaran AK: Novel role for Na,K-ATPase in phosphatidylinositol 3-kinase signaling and suppression of cell motility. Mol Biol Cell 16: 1082-1094, 2005.

20. Angelisova P, Drbal K, Horejsi V and Cerny J: Association of CD10/neutral endopeptidase 24.11 with membrane micro domains rich in glycosylphosphatidylinositol-anchored proteins and Lyn kinase. Blood 93: 1437-1439, 1999.

21. Kibbey RG, Rizo J, Gierasch LM and Anderson RG: The LDL receptor clustering motif interacts with the clathrin terminal domain in a reverse turn conformation. J Cell Biol 142: 59-67, 1998.

22. Heuser JE and Keen J: Deep-etch visualization of proteins involved in clathrin assembly. J Cell Biol 107: 877-886, 1988.

23. ter Haar E, Musacchio A, Harrison SC and Kirchhausen T: Atomic structure of clathrin: a beta propeller terminal domain joins an alpha zigzag linker. Cell 95: 563-573, 1998.

24. Scheele U, Alves J, Frank R, Duwel M, Kalthoff C and Ungewickell E: Molecular and functional characterization of clathrin- and AP-2-binding determinants within a disordered domain of auxilin. J Biol Chem 278: 25357-25368, 2003.

25. Scheele U, Kalthoff $C$ and Ungewickell E: Multiple interactions of auxilin 1 with clathrin and the AP-2 adaptor complex. J Biol Chem 276: 36131-36138, 2001.

26. Legendre-Guillemin V, Metzler M, Charbonneau M, et al: HIP1 and HIP12 display differential binding to F-actin, AP2, and clathrin. Identification of a novel interaction with clathrin light chain. J Biol Chem 277: 19897-19904, 2002.

27. Waelter S, Scherzinger E, Hasenbank R, et al: The huntingtin interacting protein HIP1 is a clathrin and alpha-adaptin-binding protein involved in receptor-mediated endocytosis. Hum Mol Genet 10: 1807-1817, 2001.

28. Laporte SA, Miller WE, Kim KM and Caron MG: ß-arrestin/ AP-2 interaction in $\mathrm{G}$ protein-coupled receptor internalization: identification of a beta-arrestin binging site in beta 2 -adaptin. J Biol Chem 277: 9247-9254, 2002.

29. Kim KM, Gainetdinov RR, Laporte SA, Caron MG and Barak LS: $\mathrm{G}$ protein-coupled receptor kinase regulates dopamine D3 receptor signaling by modulating the stability of a receptor-filamin-betaarrestin complex. A case of autoreceptor regulation. J Biol Chem 280: 12774-12780, 2005.

30. Rao DS, Hyun TS, Kumar PD, et al: Huntingtin-interacting protein 1 is overexpressed in prostate and colon cancer and is critical for cellular survival. J Clin Invest 110: 351-360, 2002. 